\title{
Factors influencing the stability of freeze-dried stress-resilient and stress-sensitive strains of bifidobacteria
}

\author{
O. F. Celik and D. J. O'Sullivan ${ }^{1}$ \\ Department of Food Science and Nutrition, Center for Microbial and Plant Genomics, University of Minnesota, 1500 Gortner Ave., St. Paul 55108
}

\begin{abstract}
Freeze-drying is a common method for preservation of probiotics, including bifidobacteria, for further industrial applications. However, the stability of freezedried bifidobacteria varies depending on the freezedrying method and subsequent storage conditions. The primary goals of this study were to develop an optimized freeze-drying procedure and to determine the effects of temperature, water activity, and atmosphere on survival of freeze-dried bifidobacteria. To address these goals, a commercially used bifidobacteria strain that is resilient to stress, Bifidobacterium animalis ssp. lactis $\mathrm{Bb}-12$, and a characterized intestinal strain that is more sensitive to stress conditions, Bifidobacterium longum DJO10A, were used. A freeze-drying protocol was developed using trehalose as the cryoprotectant, which resulted in almost no loss of viability during freeze-drying. Resuscitation medium, temperature, and time did not significantly influence recovery rates when this cryoprotectant was used. The effects of temperature $\left(-80\right.$ to $\left.45^{\circ} \mathrm{C}\right)$, water activity $(0.02$ to 0.92$)$, and atmosphere (air, vacuum, and nitrogen) were evaluated for the stability of the freeze-dried powders during storage. Freeze-dried B. animalis ssp. lactis Bb-12 was found to survive under all conditions tested, with optimum survival at temperatures up to $21^{\circ} \mathrm{C}$, water activities up to 0.44 , and all 3 atmospheres tested. The intestinal-adapted strain B. longum DJO10A was much more sensitive to the different storage conditions, but could be adequately maintained using optimum conditions. These optimum storage conditions included frozen storage, replacement of oxygen with nitrogen, and water activities between 0.11 and 0.22 . These results indicated that an optimized storage environment is required to maintain viability of stress-sensitive bifidobacteria strains, whereas stress-resilient bifidobacteria strains can maintain viability over a wide range of storage conditions, which is practical in countries where
\end{abstract}

Received October 30, 2012.

Accepted February 20, 2013.

${ }^{1}$ Corresponding author: dosulliv@umn.edu controlled cold storage conditions may not be readily available.

Key words: Bifidobacterium longum, Bifidobacterium animalis ssp. lactis, probiotic, trehalose

\section{INTRODUCTION}

The observations of Metchnikoff and Mitchell (1907) on the health benefits of ingesting lactic acid-producing bacteria laid the groundwork for the current probiotic era, which is a rapidly growing field worldwide. Dairy foods are particularly good vehicles for the delivery of probiotics to humans, as lactic acid-producing cultures are suited to this environment. The first commercial probiotic drink Yakult (Yakult Honsha Co. Ltd., Tokyo, Japan), containing Lactobacillus casei Shirota, was introduced in 1935 in Japan and is still sold today throughout the world (Fukushima and Hurt, 2011). Bifidobacteria were subsequently introduced because of their association with healthy intestines in breast-fed infants and their reduced numbers in the elderly, with a concomitant reduction in gut health. In the last 20 yr, the global probiotic market has been growing due to considerable progress in probiotic research, and sales are estimated to reach $\$ 19.6$ billion in 2013 (Granato et al., 2010). Currently, Lactobacillus and Bifidobacterium are the most common probiotic genera used in food products.

Many challenges exist in the development of probiotic-containing food products, such as selection of effective strains and their survival during processing and storage. An important component of current selection practices for bifidobacteria for use as probiotics is their ability to survive food processing and storage. Their viability has been a technological issue for food manufacturers, particularly in fermented foods such as yogurt. As bifidobacteria are largely obligate anaerobic bacteria and not very acid tolerant, they are less stable in yogurts compared with lactobacilli (El-Dieb et al., 2012). Hence, the viability of bifidobacteria becomes an important issue and until technological advancements are made in protecting their viability in foods, strains are selected primarily for this feature rather than the 
myriad other characteristics that pertain to their probiotic functioning in the gut.

Drying cultures can enable their long-term storage and transportation without the need for refrigeration, if conditions are optimized. However, conditions are not the same for all cultures, with some, particularly many bifidobacteria cultures, being particularly sensitive (Meng et al., 2008). Although dried cultures have advantages during shipping and storage, critical parameters affect viability following their incorporation into foods, such as the nature of the food matrix, rehydration temperature, rehydration time, and powder-to-liquid ratios (Champagne, 2009). Freezedrying and spray-drying are the 2 methods currently used to dry probiotics, with spray-drying being the preferred choice because of its cost effectiveness. In terms of viability, freeze-drying is the better process but its cost has hindered its use in large-scale processes. Spray-drying can be used for some cultures, but the conditions require optimization because probiotics are sensitive to heat (Chávez and Ledeboer, 2007). Studies have confirmed that bifidobacteria in general are very sensitive to spray-drying and showed superior survival rates following freeze-drying in different media (Wang et al., 2004; Chávez and Ledeboer, 2007; Wong et al., 2010). Based on these findings, freeze-drying became a popular method of stabilizing bifidobacteria before incorporation into food products.

Maintaining viability not only during the freezedrying process but also during storage is a critical challenge for commercial production of bifidobacteria for probiotic applications (Saarela et al., 2005). Cryoprotectants such as polymers and sugars have been involved in the freeze-drying process to improve the survival rate of bifidobacteria (Kiviharju et al., 2005; Saarela et al., 2005). However, the abilities to survive during freeze-drying and subsequent storage are not linked. Therefore, factors that affect survival during storage need to be defined (Champagne et al., 2005). Storage temperature, exposure to oxygen, and water activity are several critical factors that affect viability of dried probiotics (Chávez and Ledeboer, 2007). Only a few studies show the detrimental effects of storage temperature and water activity on freeze-dried bifidobacteria (Champagne et al., 1996; Bruno and Shah, 2003; Abe et al., 2009a).

Although bifidobacteria with high stress tolerance are commonly used in food products, there is interest in using other less-tolerant strains that may be more suited to competing in the gut, provided that suitable conditions are developed to maintain their viability in foods. The objectives of this study were to develop a freeze-drying protocol for bifidobacteria, including stress-sensitive strains, and to evaluate the effects of temperature, water activity, and atmosphere during subsequent storage of the freeze-dried powders. Two potential probiotic bifidobacteria with different stress tolerances were used to achieve these objectives. One strain represented the most stress-adapted group of bifidobacteria, Bifidobacterium animalis ssp. lactis (Simpson et al., 2005), and the other strain, Bifidobacterium longum DJO10A, a characterized intestinal strain with minimum pure culture adaptation, represented a highly stress-sensitive group of bifidobacteria.

\section{MATERIALS AND METHODS}

\section{Bacterial Strains, Growth Media, and Culture Growth Conditions}

Two strains of bifidobacteria were used for this study: B. animalis ssp. lactis $\mathrm{Bb}-12$ and B. longum ssp. longum DJO10A. Strain Bb-12, a common commercial probiotic used in many food products, was obtained from Chr. Hansen Inc. (Milwaukee, WI). Strain DJO10A is an intestinal strain that was isolated and characterized in our laboratory (Islam, 2006). Cultures were used from frozen stocks stored at $-80^{\circ} \mathrm{C}$ in de Man, Rogosa, and Sharpe medium (MRS, BD Biosciences, San Jose, CA) containing $15 \%$ glycerol. Cultures were cultivated in MRS and incubated at $37^{\circ} \mathrm{C}$ anaerobically. A selective medium for bifidobacteria, bifidobacteria iodoacetate selective medium (BIM-25; Muñoa and Pares, 1988), was used, consisting of $3.8 \%$ reinforced clostridial agar (Oxoid, Basingstoke, UK), $0.005 \%$ L-cysteine $\cdot \mathrm{HCl}$, $0.002 \%$ nalidixic acid, $0.00085 \%$ polymyxin B sulfate, $0.005 \%$ kanamycin sulfate, $0.0035 \%$ iodoacetic acid, $0.0025 \% 2,3,5$ triphenyl tetrazolium chloride, and $1.8 \%$ agar.

\section{Preparation of Cultures for Freeze-Drying}

Strains of Bb-12 and DJO10A were inoculated from stock cultures into tubes of MRS containing $0.05 \%$ Lcysteine $\cdot \mathrm{HCl}$ and incubated anaerobically at $37^{\circ} \mathrm{C}$ for $1 \mathrm{~d}$. Each culture was subinoculated at $2 \%$ into $4 \mathrm{~L}$ of $\mathrm{MRS}+0.05 \% \mathrm{~L}$-cysteine. $\mathrm{HCl}$ and incubated anaerobically at $37^{\circ} \mathrm{C}$. Optical density at $600 \mathrm{~nm}\left(\mathbf{O D}_{600}\right)$ was checked for determination of growth kinetics; when an $\mathrm{OD}_{600}$ of 1.0 was reached, the freeze-drying procedure was applied.

\section{Preparation of Freeze-Drying Buffers}

Sodium phosphate buffer $(\mathrm{pH}=6.8)$ was used as the base buffer after autoclaving at $121^{\circ} \mathrm{C}$ for $15 \mathrm{~min}$. The base buffer was supplemented with dried skim milk (DSM; Difco), trehalose, sucrose, or combinations 
thereof, as cryoprotectants. Four different buffers were tested to observe their effects on the survival of bifidobacteria after freeze-drying: (1) sodium phosphate buffer; (2) sodium phosphate buffer $+5 \%$ DSM; (3) sodium phosphate buffer $+5 \%$ DSM $+4 \%$ trehalose; and (4) sodium phosphate buffer $+5 \%$ DSM $+4 \%$ trehalose $+5 \%$ sucrose.

\section{Freeze-Drying Procedure}

Previous procedures (Bruno and Shah, 2003; Saarela et al., 2006) were used to develop a suitable freezedrying procedure for this study. Cultures were grown in MRS $+0.05 \%$ L-cysteine. $\mathrm{HCl}$ as described above and $\mathrm{OD}_{600}$ was checked periodically. When the $\mathrm{OD}_{600}$ was 1.0, the following additional steps were applied to both cultures.

Two 1-mL aliquots of culture were taken for subsequent triplicate arbitrarily primed (TAP)-PCR analysis and enumeration of viable cell counts after serial dilution. The remaining culture was divided into $250-\mathrm{mL}$ centrifuge tubes and centrifuged at 4,500 $\times g$ for $15 \mathrm{~min}$ at $4^{\circ} \mathrm{C}$ and the supernatant was discarded. The pellets in each tube were resuspended with $20 \mathrm{~mL}$ of $0.1 \mathrm{M}$ sodium phosphate buffer and transferred to 50-mL Falcon tubes. Tubes were centrifuged at 2,792 $\times$ $g$ for 15 min at $4^{\circ} \mathrm{C}$ and the supernatant was discarded. The pellets in each tube were resuspended with $10 \mathrm{~mL}$ of 1 of the 4 freeze-drying buffers and transferred to new 50-mL Falcon tubes. The tubes containing resuspended cells were sealed with parafilm and aluminum foil and were frozen at $-80^{\circ} \mathrm{C}$ overnight. The cultures were then dried in a freeze-dryer (Labconco FreeZone 6 L, Labconco Corp., Kansas City, MO) set to dry at $-55^{\circ} \mathrm{C}$ and $1.8 \mathrm{~Pa}$ for $45 \mathrm{~h}$.

\section{Preparation of Resuscitation Media}

Three types of media were tested for the selection of optimum resuscitation medium: molecular-grade water, MRS $+0.05 \%$ L-cysteine. $\mathrm{HCl}$, and MRS $+0.05 \%$ Lcysteine $\cdot \mathrm{HCl}$ supplemented with 5\% DSM.

\section{Viable Plate Counts}

Before Freeze-Drying. Cultures were serially diluted (1 in $9 \mathrm{~mL})$ in MRS $+0.05 \%$ L-cysteine $\cdot \mathrm{HCl}$ medium up to $10^{-8}$ followed by spread-plating on fresh agar plates of the same medium. Selected dilutions were also plated on BIM-25 agar for verification. All plates were incubated anaerobically at $37^{\circ} \mathrm{C}$ for $2 \mathrm{~d}$.

After Freeze-Drying. The equivalent quantity of freeze-dried powders required for viable cell counts was determined based on the total volume of culture that had been used for freeze-drying and the amount of powder gained after freeze-drying. Freeze-dried powder was resuspended in $10 \mathrm{~mL}$ of 1 of 3 resuscitation media by vortexing for approximately $1 \mathrm{~min}$. This culture was then serially diluted (1 in $9 \mathrm{~mL}$ ) in fresh MRS + 0.05\% L-cysteine. $\mathrm{HCl}$ media and followed by spread-plating on fresh agar plates of the same medium. All plates were incubated anaerobically at $37^{\circ} \mathrm{C}$ for $2 \mathrm{~d}$.

\section{DNA Fingerprinting of Cultures}

To ensure the purity of the cultures before and after freeze-drying, a DNA fingerprint was obtained before and after culture preparations. A fingerprinting procedure that had this capability was TAP-PCR and it was carried out essentially as described in Cusick and O'Sullivan (2000). Specifically, $1.5 \mathrm{~mL}$ of overnight broth culture was pelleted and resuspended in 200 $\mu \mathrm{L}$ of molecular-grade water. Cells were disrupted by agitation with 0.5 volume of glass beads $(<106 \mu \mathrm{m}$ in diameter; Sigma, St. Louis, MO) in a Minibeadbeater-8 (Biospec Products, Bartlesville, OK) for $30 \mathrm{~s}$. After a 10-fold dilution, $2 \mu \mathrm{L}$ was used as the DNA template. The reaction was performed using $100 \mathrm{pmol}$ of the primer P32 (5'-CAGCAGCCGCGGTAATWC), which has homology to a universally conserved region of the 16S rRNA gene, $1 \mu \mathrm{L}$ of Taq polymerase (New England Biolabs, Ipswich, MA), $5 \mu \mathrm{L}$ of the reaction buffer (New England Biolabs), and $0.2 \mathrm{~m} M$ dNTP (Promega, Madison, WI) in a final volume of $50 \mu \mathrm{L}$. The PCR mixtures were overlaid with $25 \mu \mathrm{L}$ of mineral oil. Amplification was carried out using a Robocycler Gradient Thermocycler (Agilent Technologies, Santa Clara, CA) with the following conditions: 1 cycle of $94^{\circ} \mathrm{C}$ for $5 \mathrm{~min}$, followed by 40 cycles of $94^{\circ} \mathrm{C}$ for $1 \mathrm{~min}$ and 38, 40, and $42^{\circ} \mathrm{C}$ for $1 \mathrm{~min}$, followed by $72^{\circ} \mathrm{C}$ for $2 \mathrm{~min}$, followed by a final extension step at $72^{\circ} \mathrm{C}$ for $10 \mathrm{~min}$.

\section{Storage Conditions}

Three different storage conditions were evaluated. The first evaluated the effect of different temperatures. Freeze-dried Bb-12 and DJO10A powders were stored at different temperatures $(-80,-20,4,21,37$, and $45^{\circ} \mathrm{C}$ ) and viability was monitored periodically using viable cell counts.

The second parameter examined was water activity $\left(\mathbf{a}_{\mathrm{w}}\right)$. Freeze-dried cultures of bifidobacteria were stored at 9 different water activity conditions ranging from 0.02 to 0.92. Saturated salt solutions were used to provide the indicated water activities as follows: calcium chloride $\left(\mathrm{a}_{\mathrm{w}}=0.02\right)$, lithium chloride $\left(\mathrm{a}_{\mathrm{w}}=0.11\right)$, potassium acetate $\left(a_{\mathrm{w}}=0.22\right)$, magnesium chloride $\left(\mathrm{a}_{\mathrm{w}}=0.32\right)$, potassium carbonate $\left(\mathrm{a}_{\mathrm{w}}=0.44\right)$, magnesium nitrate 
$\left(\mathrm{a}_{\mathrm{w}}=0.50\right)$, sodium acetate $\left(\mathrm{a}_{\mathrm{w}}=0.67\right)$, ammonium sulfate $\left(\mathrm{a}_{\mathrm{w}}=0.79\right)$, and sodium phosphate dibasic $\left(\mathrm{a}_{\mathrm{w}}\right.$ $=0.92$; Gopalakrishna and Prabhakar, 1983). Freezedried powders were spread over shallow containers and placed in mason jars filled with saturated salt solutions. All jars were stored at room temperature $\left(21^{\circ} \mathrm{C}\right)$.

The third parameter examined was atmospheric condition. Normal air, nitrogen-based anaerobic atmosphere, and vacuum (to represent the absence of an atmosphere) were applied. Freeze-dried powders were placed in anaerobic jars and jars were immediately closed tightly. Air was evacuated by vacuum. A nitrogen anaerobic atmosphere was provided by pumping nitrogen inside the jar after all the air was evacuated. All jars were stored at room temperature $\left(21^{\circ} \mathrm{C}\right)$. Samples were taken at indicated intervals for analysis of culture viability. All samples were analyzed in triplicate and data were statistically analyzed using Excel (Microsoft Corp., Redmond, WA).

\section{RESULTS}

\section{Selection of Optimum Cryoprotectant and Resuscitation Medium}

Four cryoprotectants and 3 resuscitation media were tested in combination to determine the optimum cryoprotectant and resuscitation medium. Bifidobacterium longum DJO10A was used as the model culture for selection of the optimum cryoprotectant and resuscita- tion medium because it was previously shown to be more sensitive to processing conditions compared with B. animalis ssp. lactis $\mathrm{Bb}-12$ (Scheller and O'Sullivan, 2011). The highest recovery rate was obtained when sodium phosphate buffer $+5 \%$ DSM $+4 \%$ trehalose was used as the cryoprotectant, regardless of the resuscitation media (Figure 1). Although MRS $+0.05 \%$ Lcysteine $\cdot \mathrm{HCl}+5 \% \mathrm{DSM}$ had a slightly higher recovery rate, the choice of resuscitation medium did not significantly influence recovery rates when this cryoprotectant was used. However, MRS $+0.05 \%$ L-cysteine $\cdot \mathrm{HCl}$ gave better recovery rates in general. Therefore, sodium phosphate buffer $+5 \%$ DSM $+4 \%$ trehalose was selected as the optimum cryoprotectant and MRS + $0.05 \% \mathrm{~L}$-cysteine $\cdot \mathrm{HCl}$ was selected as the resuscitation medium for further freeze-drying applications.

\section{Evaluation of Resuscitation Time and Temperature for Freeze-Dried Bifidobacteria in Milk}

Freshly freeze-dried powders of strain DJO10A and $\mathrm{Bb}-12$ were resuscitated in sterilized skim milk that had initial temperatures of 4 and $21^{\circ} \mathrm{C}$. Viable cell counts were carried out and recorded as " 0 h." The tubes were then stored at $4^{\circ} \mathrm{C}$ and $21^{\circ} \mathrm{C}$ and viable cell counts were enumerated at $1,2,3$, and $6 \mathrm{~h}$ to observe the effect of different temperatures on the resuscitation times of freeze-dried cultures of bifidobacteria in milk. Resuscitation time and temperature did not increase the viability of DJO10A or Bb-12 (Figure 2). However, extended

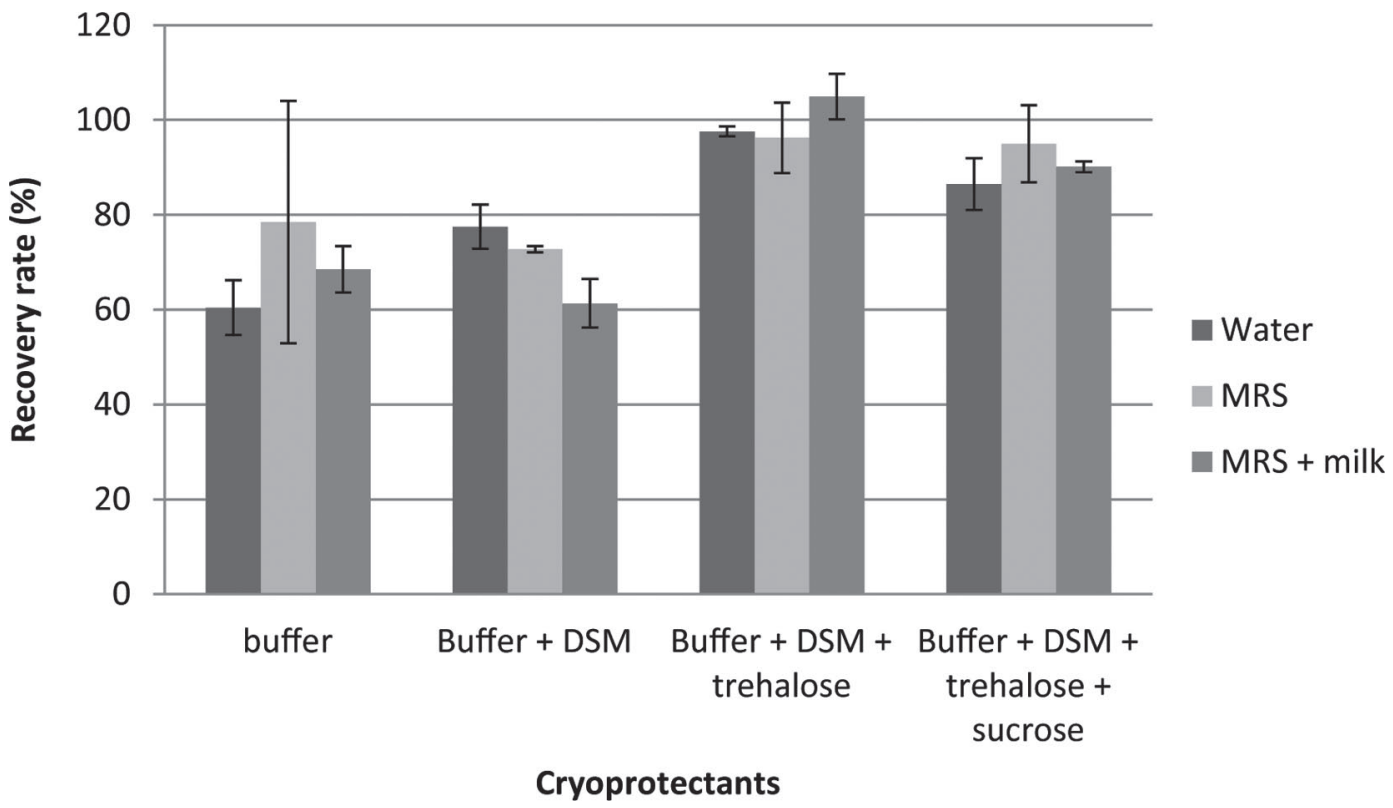

Figure 1. Recovery rates (\%) of Bifidobacterium longum DJO10A in different resuscitation media after freeze-drying with different cryoprotectants. DSM = dried skim milk; MRS = de Man, Rogosa, and Sharpe medium. Each bar represents the average of 3 replicates and the error bars show standard deviations. 
a)



b)

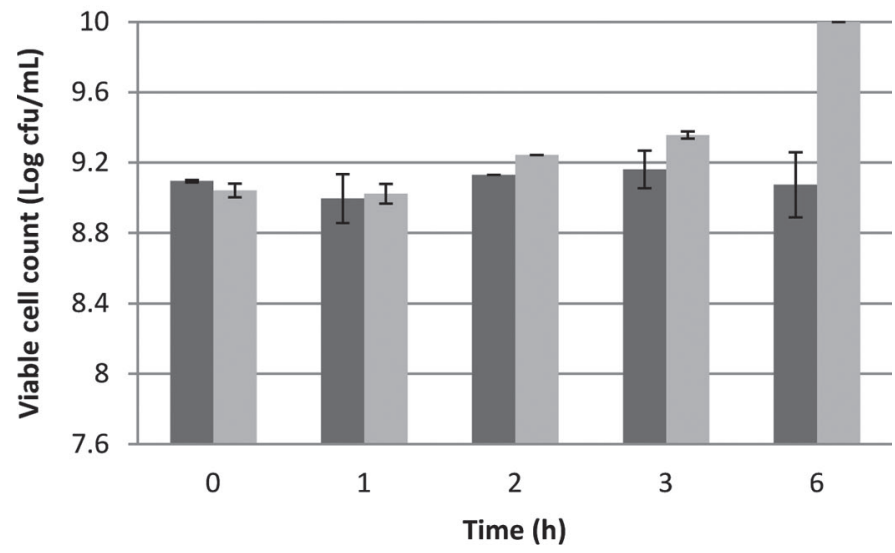

Figure 2. Resuscitation time in milk at 4 or $21^{\circ} \mathrm{C}$ for (a) Bifidobacterium longum DJO10A, and (b) Bifidobacterium animalis ssp. lactis Bb-12. Each bar represents the average of 3 replicates, and the error bars show standard deviations.

resuscitation for $6 \mathrm{~h}$ at $21^{\circ} \mathrm{C}$ reduced the viability of strain DJO10A, whereas strain Bb-12 resumed growing and increased in numbers.

\section{DNA Fingerprints of Freeze-Dried Cells}

A TAP-PCR DNA fingerprint demonstrated that both strains had distinctive fingerprints and the "before" and "after" fingerprints were identical (Figure 3). Therefore, based on this fingerprinting analysis, no detectable contamination or chromosomal damage occurred during the freeze-drying procedure.

\section{Effect of Temperature on Survival of Freeze-Dried Bifidobacteria During Storage}

The recovery rate for strain DJO10A was significantly affected by storage temperature. In general, the recovery rate of DJO10A powders decreased as the storage temperature increased above freezing (Figure 4a). The highest viability was obtained under frozen storage conditions, in which no considerable loss of viability was observed, even after 10 mo. Freeze-dried powders of strain Bb-12 did not show a significant reduction in viability during $10 \mathrm{mo}$ of storage up to $21^{\circ} \mathrm{C}$ (Figure $4 \mathrm{~b})$. The greatest reduction in viability (about $3 \operatorname{logs}$ ) occurred after $10 \mathrm{mo}$ at 37 and $45^{\circ} \mathrm{C}$.

\section{Effect of Water Activity on Survival of Freeze-Dried Bifidobacteria During Storage}

Freeze-dried powders that were stored at different water activities had markedly different physical properties. Powder properties ranged from dusty to sticky as water activity increased, and powders stored at $\mathrm{a}_{\mathrm{w}}=$ 0.92 became a mixture of powder and water. The colors

\section{Before}
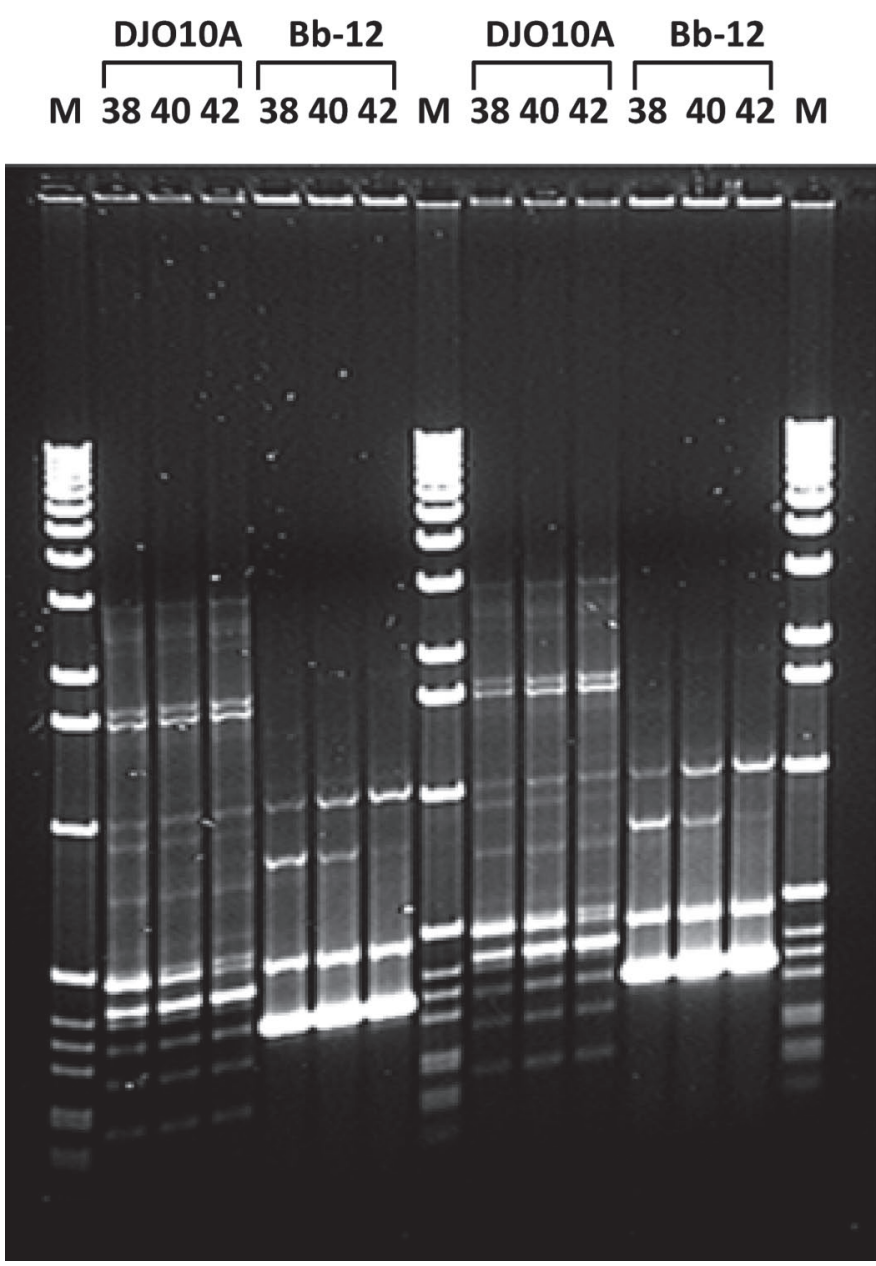

Figure 3. Triplicate arbitrarily primed-PCR DNA fingerprints of Bifidobacterium longum DJO10A and Bifidobacterium animalis ssp. lactis Bb-12 cell batches before and after freeze-drying. Numbers above the lanes represent annealing temperatures $\left({ }^{\circ} \mathrm{C}\right) ; \mathrm{M}=1-\mathrm{kb}$ DNA ladder (Invitrogen, Carlsbad, CA). 


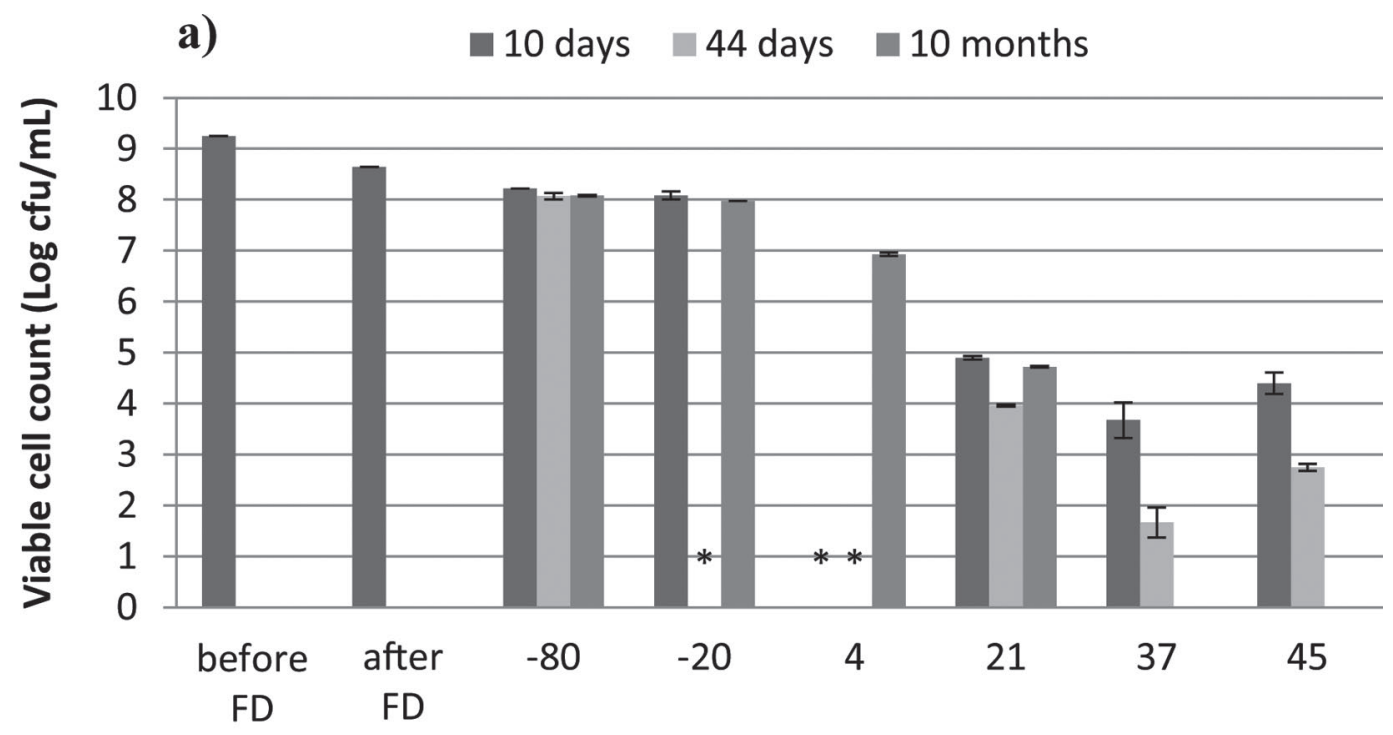

Storage temperature $\left({ }^{\circ} \mathrm{C}\right)$

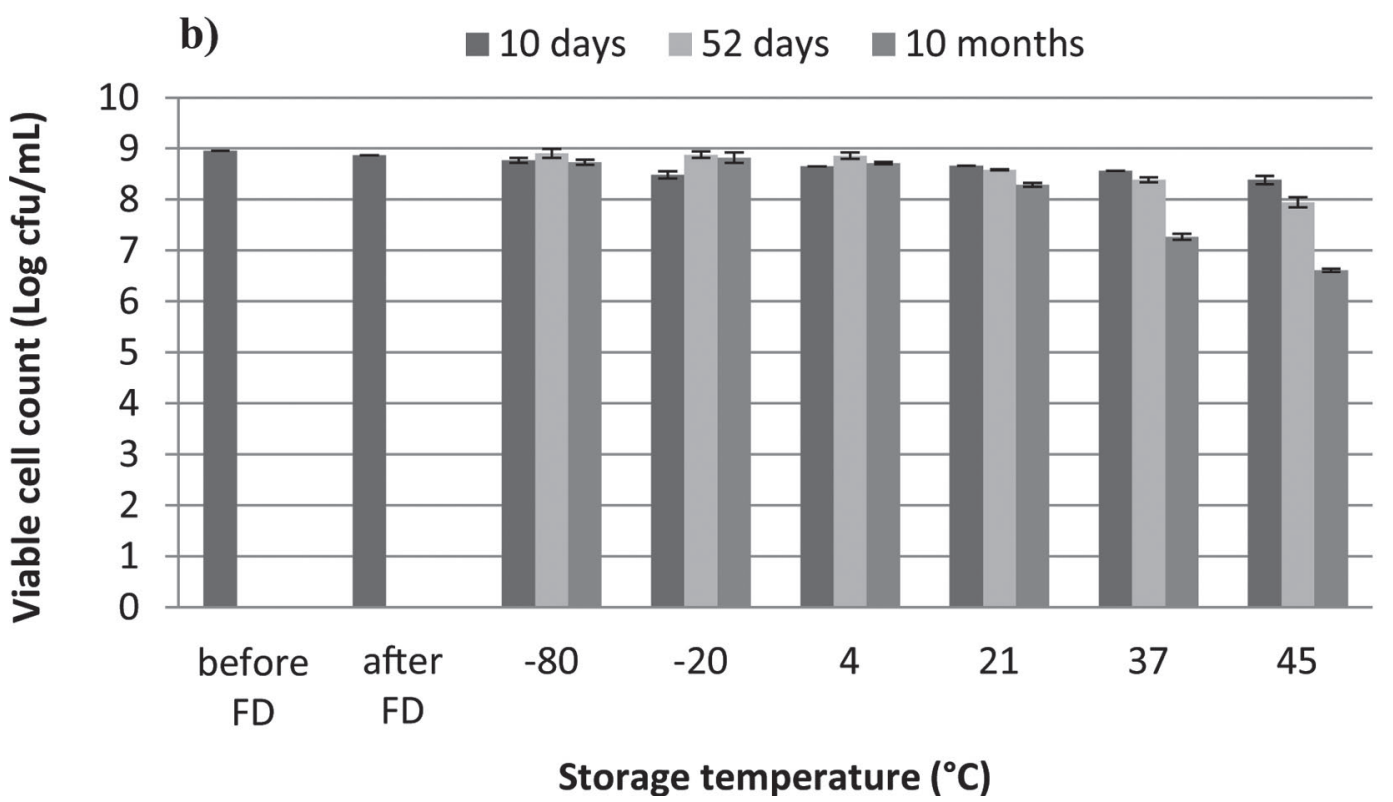

Figure 4. Viable cell counts for (a) Bifidobacterium longum DJO10A, and (b) Bifidobacterium animalis ssp. lactis Bb-12 stored at different temperatures and different periods. Each bar represents the average of 3 replicates, and the error bars show standard deviations. Asterisks $(*)$ indicate undetermined viable cell counts due to technical problems. FD = freeze-drying.

of the powders also changed, ranging from cream and yellow to orange and brown (Figure 5).

Freeze-dried powders of DJO10A that were stored at higher water activities (0.67 to 0.92 ) could not be dissolved completely in the resuscitation medium, which prevented an accurate viable cell count from being done. Although similar effects were observed for Bb12 freeze-dried powders, viable plate counts could be performed for all conditions except $\mathrm{a}_{\mathrm{w}}=0.92$. After $45 \mathrm{~d}$ of storage, visible molds started to appear on the freeze-dried powders stored at $\mathrm{a}_{\mathrm{w}}=0.92$. Therefore, no more viable cell counts were performed after $45 \mathrm{~d}$ for either culture at this water activity.

The viability of freeze-dried DJO10A decreased at different rates during storage at different water activities. After $46 \mathrm{~d}$ of storage, powders stored at $\mathrm{a}_{\mathrm{w}}=0.11$ and 0.22 had the highest viability, whereas those stored at $\mathrm{a}_{\mathrm{w}}=0.44$ and 0.5 had the lowest viability (Figure $6 a)$. The viability of freeze-dried BB-12 was much higher than that of DJO10A over storage time. Its viability 




Figure 5. Physical appearance of freeze-dried cultures of Bifidobacterium longum DJO10A with different water activities after $10 \mathrm{~d}$. Color version is available in the online PDF version.

did not change significantly over 10 mo of storage at conditions of $\mathrm{a}_{\mathrm{w}}=0.44$ or less. Although viability was still very high up to $50 \mathrm{~d}$ at higher water activities, no viable cells were detected after 10 mo of storage for $\mathrm{a}_{\mathrm{w}}$ $=0.67$ or higher. After $10 \mathrm{mo}$, the viability of freezedried Bb-12 stored at $\mathrm{a}_{\mathrm{w}}=0.5$ decreased significantly (almost $3 \operatorname{logs}$ ) and no accurate viable cell counts could be obtained for higher water activities due to solubility problems (Figure 6b).

\section{Effect of Atmospheric Conditions on Survival of Freeze-Dried Bifidobacteria During Storage}

After 10 mo of storage, no viable cells could be detected for DJO10A under vacuum or air at room temperature, as determined by no growth of the resuscitated powders on MRS agar plates (Figure 7a). Temperature was the main factor affecting viability but it was clear that exposure to either air or vacuum had a detrimental effect on viability of DJO10A. No significant difference was found in viability of DJO10A stored under air and nitrogen for up to $44 \mathrm{~d}$. However, nitrogen was found to be the most suitable atmosphere for extended storage of freeze-dried DJO10A. The nitrogen atmosphere caused about a 5-log reduction in viability during the first $10 \mathrm{~d}$ but viability was kept constant after that up to 10 mo. The effects of different atmospheric conditions on the viability of freeze-dried Bb-12 did not differ and almost no loss of viability was observed (Figure 7b).

\section{DISCUSSION}

Maintaining the viability of probiotics is an important task for food manufacturers because viability is important for probiotics to provide health benefits (FAO/WHO, 2001). Thus, the freeze-drying process is commonly used to preserve and store probiotics for industrial applications. In this study, a freeze-drying procedure using trehalose as the cryoprotectant was developed and the effects of various temperatures, water activities, and atmospheres on the stability of a freeze-dried commercial strain and a minimally cultured intestinal strain of bifidobacteria during storage were investigated.

The freeze-drying process can cause cellular damage because of the aqueous nature of the contents. Hence, sugars are widely used as cryoprotectants, with nonreducing disaccharides with high glass transition temperatures $\left(\mathrm{T}_{\mathrm{g}}\right)$ shown to provide higher viability for 

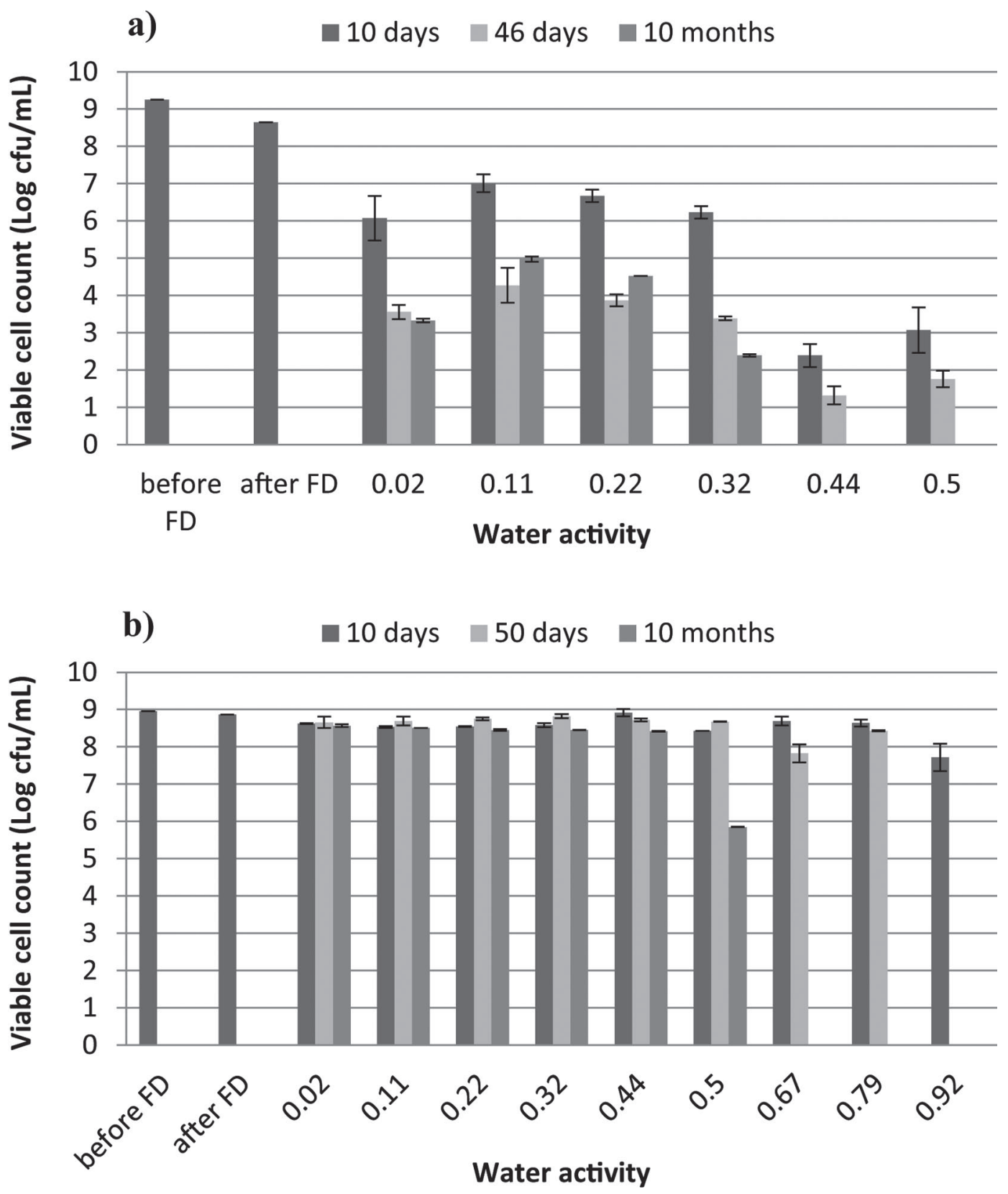

Figure 6. Viable cell counts for (a) Bifidobacterium longum DJO10A, and (b) Bifidobacterium animalis ssp. lactis Bb-12 stored at different water activities $\left(\mathrm{a}_{\mathrm{w}}\right.$; values shown in each panel). $\mathrm{FD}=$ freeze-drying. Each bar represents the average of 3 replicates, and the error bars show standard deviations.

freeze-dried probiotics (Zayed and Roos, 2004; Miao et al., 2008; Siaterlis et al., 2009). Our study established the cryoprotective effect of trehalose, a nonreducing disaccharide, as an effective cryoprotectant for freezedrying of bifidobacteria.

Cells injured during freeze-drying can be recovered by applying suitable resuscitation conditions, such as resuscitation medium, time, and temperature (Carvalho et al., 2004). Variable recovery rates were found depending on the cryoprotective agents used in this study. Similar values were obtained even when molecular-grade water was used as the resuscitation medium. These results suggest that the effect of selected resuscitation media on the recovery rate of freeze-dried $B$. longum DJO10A is not significant and that it depends more on the cryoprotective agent used.

Confirmation of strain identity is another key point to ensure contamination does not occur during the 

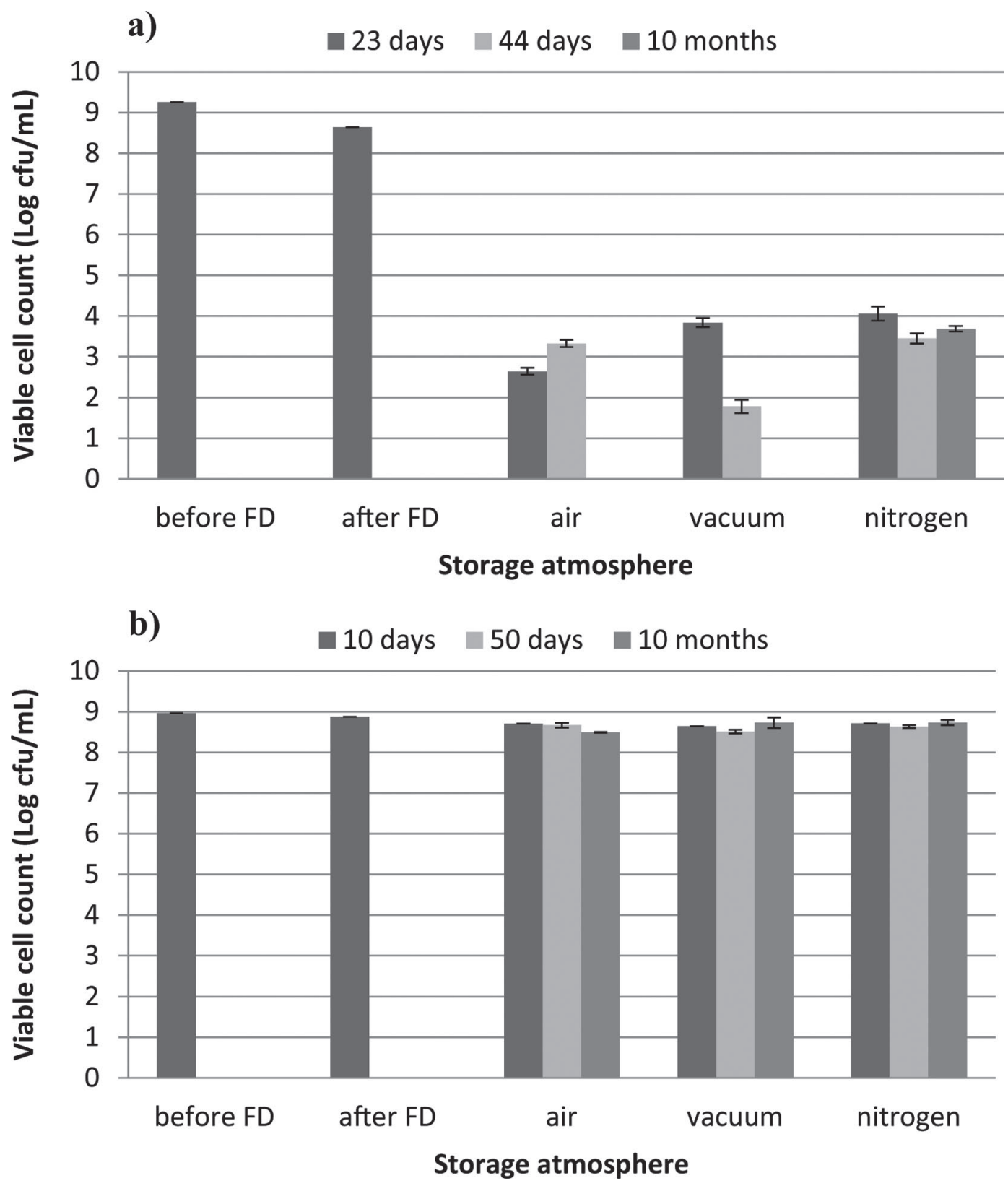

Figure 7. Viable cell counts for (a) Bifidobacterium longum DJO10A, and (b) Bifidobacterium animalis ssp. lactis Bb-12 stored at different atmospheres at $21^{\circ} \mathrm{C}$. FD $=$ freeze-drying. Each bar represents the average of 3 replicates, and the error bars show standard deviations.

freeze-drying process. Triplicate arbitrarily primedPCR DNA fingerprinting has previously been known to be a convenient method for differentiation of bifidobacteria profiles (Cusick and O'Sullivan, 2000) and was in this study used to ensure the purity of cultures before and after freeze-drying.

Higher storage temperatures yielded lower recovery rates for strain DJO10A, which is consistent with findings for other bifidobacteria freeze-dried powders (Champagne et al., 1996; Bruno and Shah, 2003; Abe et al., 2009b). Freeze-dried powders of the commercial probiotic Bb-12 were much more resilient to high storage temperatures: viability was constant even after 10 mo of storage at room temperature. The 3 log reduction in viability of $\mathrm{Bb}-12$ at $45^{\circ} \mathrm{C}$ is manageable for storage in tropical countries where storage temperatures might be difficult to control.

After 10 mo of storage at room temperature, viability was protected only at $\mathrm{a}_{\mathrm{w}}<0.44$, with an optimum of 0.11 for freeze-dried DJO10A powder. Viability of DJO10A 
decreased as $\mathrm{a}_{\mathrm{w}}$ increased or decreased from 0.11. These results correlate with the findings of other studies, in which greater stability of strains of $B$. longum is associated with lower water activity (Nagawa et al., 1988; Abe et al., 2009a). However, strain Bb-12 was found to be more tolerant to different water activity conditions. Although Chávez and Ledeboer (2007) suggested the use of $\mathrm{a}_{\mathrm{w}}<0.25$ for better survival of freeze-dried Bb-12, based on our results, Bb-12 can be stored at $\mathrm{a}_{\mathrm{w}}$ up to 0.44 without any loss of viability. This difference may be ascribed to the different freeze-drying procedures applied and the cryoprotectants used in this study. Despite the fact that the commercial isolate Bb-12 can tolerate a wider range of water activity conditions without any loss in viability, this study demonstrated that the viability of sensitive cultures, such strain DJO10A, can be maintained when cultures are stored under controlled water activity conditions.

Water activity was found to be an important factor affecting not only the stability but also the physical properties of freeze-dried bifidobacteria, such as solubility and color. Solubility problems and color change at higher water activities might be associated with the occurrence of various types of nonenzymatic browning reactions occurring, including the Maillard reaction (Stapelfeldt et al., 1997; Kurtmann et al., 2009). Trehalose has no role in these reactions because it is a nonreducing sugar, but lactose from the DSM may have contributed to such reactions. Although loss of solubility at high water activities was a problem for both strains, it was much more pronounced for DJO10A cells, which may be due to the tendency of this strain to form clumps in broth media.

Nitrogen was the only atmosphere that protected the viability of freeze-dried DJO10A in storage for up to 10 mo. To prevent detrimental effects of oxygen, Chávez and Ledeboer (2007) suggested storage under vacuum or replacing oxygen with nitrogen for the storage of freeze-dried bifidobacteria. All storage conditions were comparable for the commercial strain Bb-12, consistent with its superior tolerance to stress conditions.

Bifidobacteria are anaerobic bacteria but their oxygen tolerance varies from very low (e.g., B. longum) to high (e.g., B. animalis ssp. lactis; Simpson et al., 2005). When we tested the effect of temperature, freeze-dried cultures were simultaneously exposed to air, which was similar to the experimental design for testing the effect of air on storage. No loss of viability was observed for Bb-12 in either test. However, results for DJO10A were found to be distinctly different where 4 logs remained after $10 \mathrm{mo}$ of storage in the room temperature test but no viable cells remained after 10 mo of storage in the test for the effect of air on storage at the same temperature. This might be explained by a greater exposure to air when testing the effect of atmosphere, because the caps were removed from the storage tubes inside the anaerobic jars. In the temperature experiment, the storage tubes remained capped. In this study, the survival rate of freeze-dried DJO10A was highly dependent on the amount of air, but the amount of air had no influence on the survival of freeze-dried Bb-12.

\section{CONCLUSIONS}

This study demonstrated that trehalose provided the highest level of protection during freeze-drying of bifidobacteria compared with the other cryoprotectants tested. We verified the resilience of freeze-dried Bb-12 powders to a wide range of storage conditions, which makes this strain an attractive and common probiotic used in the food industry. We also determined suitable storage conditions for sensitive strains such as DJO10A. Frozen storage, replacement of oxygen with nitrogen, and water activities controlled between 0.11 and 0.22 were the optimal conditions for storage of freeze-dried bifidobacteria powders.

\section{ACKNOWLEDGMENTS}

This research was funded in part by the Dairy Management Inc./Dairy Research Institute (Rosemont, IL). Ju-Hoon Lee (University of Minnesota, St. Paul) is acknowledged for discussions on optimizing freeze-drying conditions for bifidobacteria.

\section{REFERENCES}

Abe, F., H. Miyauchi, A. Uchijima, T. Yaeshima, and K. Iwatsuki. 2009a. Effects of storage temperature and water activity on the survival of bifidobacteria in powder form. Int. J. Dairy Technol. 62:234-239.

Abe, F., H. Miyauchi, A. Uchijima, T. Yaeshima, and K. Iwatsuki. 2009b. Stability of bifidobacteria in powdered formula. Int. J. Food Sci. Technol. 44:718-724.

Bruno, F. A., and N. P. Shah. 2003. Viability of two freeze-dried strains of Bifidobacterium and of commercial preparations at various temperatures during prolonged storage. J. Food Sci. 68:2336-2339.

Carvalho, A. S., J. Silva, P. Ho, P. Teixeira, F. X. Malcata, and P. Gibbs. 2004. Relevant factors for the preparation of freeze-dried lactic acid bacteria. Int. Dairy J. 14:835-847.

Champagne, C. P. 2009. Some technological challenges in the addition of probiotic bacteria to foods. Pages 761-804 in Prebiotics and Probiotics Science and Technology. D. Charalampopoulos and R. A. Rastall, ed. Springer, New York, NY.

Champagne, C. P., N. Gardner, and D. Roy. 2005. Challenges in the addition of probiotic cultures to foods. Crit. Rev. Food Sci. Nutr. 45:61-84.

Champagne, C. P., F. Mondou, Y. Raymond, and D. Roy. 1996. Effect of polymers and storage temperature on the stability of freezedried lactic acid bacteria. Food Res. Int. 29:555-562.

Chávez, B. E., and A. M. Ledeboer. 2007. Drying of probiotics: Optimization of formulation and process to enhance storage survival. Drying Technol. 25:1193-1201.

Cusick, S. M., and D. J. O'Sullivan. 2000. Use of a single, triplicate arbitrarily primed-PCR procedure for molecular fingerprinting of lactic acid bacteria. Appl. Environ. Microbiol. 66:2227-2231. 
El-Dieb, S. M., F. H. R. Abd Rabo, S. M. Badran, A. M. Abd ElFattah, and F. M. F. Elshaghabee. 2012. The growth behaviour and enhancement of probiotic viability in bioyoghurt. Int. Dairy J. $22: 44-47$.

FAO/WHO. 2001. Health and nutritional properties of probiotics in food including powder milk with live lactic acid bacteria. Food and Agriculture Organization of the United Nations and World Health Organization (FAO/WHO), Cordoba, Argentina.

Fukushima, Y., and E. Hurt. 2011. Probiotics health claims in Japan and Europe. Pages 253-281 in Lactic Acid Bacteria and Bifidobacteria: Current Progress in Advanced Research. K. Sonomoto, and A. Yokota, ed. Caister Academic Press, Norfolk, UK.

Gopalakrishna, A. G., and J. V. Prabhakar. 1983. Effect of water activity on autoxidation of raw peanut oil. J. Am. Oil Chem. Soc. 60:968-970.

Granato, D., G. F. Branco, F. Nazzaro, A. G. Cruz, and J. A. F. Faria. 2010. Functional foods and nondairy probiotic food development: Trends, concepts, and products. Comp. Rev. Food Sci. Food Safety 9:292-302.

Islam, A. 2006. Iron reversible inhibition by bifidobacteria and microbial diversity of the human intestine. MS Thesis. University of Minnesota, St. Paul.

Kiviharju, K., M. Leisola, and T. Eerikäinen. 2005. Optimization of a Bifidobacterium longum production process. J. Biotechnol. 117:299-308.

Kurtmann, L., L. H. Skibsted, and C. U. Carlsen. 2009. Browning of freeze-dried probiotic bacteria cultures in relation to loss of viability during storage. J. Agric. Food Chem. 57:6736-6741.

Meng, X. C., C. Stanton, G. F. Fitzgerald, C. Daly, and R. P. Ross 2008. Anhydrobiotics: The challenges of drying probiotic cultures. Food Chem. 106:1406-1416.

Metchnikoff, E., and P. C. Mitchell. 1907. The prolongation of life: Optimistic studies. G. P. Putnam's Sons, New York, NY. Republished in 2004 by Springer, New York, NY.

Miao, S., S. Mills, C. Stanton, G. Fitzgerald, Y. Roos, and R. Ross. 2008. Effect of disaccharides on survival during storage of freezedried probiotics. J. Dairy Sci. Technol. 88:19-30.

Muñoa, F. J., and R. Pares. 1988. Selective medium for isolation and enumeration of Bifidobacterium spp. Appl. Environ. Microbiol. $54: 1715-1718$
Nagawa, M., A. Nakabayashi, and S. Fujino. 1988. Preparation of the bifidus milk powder. J. Dairy Sci. 71:1777-1782.

Saarela, M., I. Virkajärvi, H. L. Alakomi, T. Mattila-Sandholm, A. Vaari, T. Suomalainen, and J. Mättö. 2005. Influence of fermentation time, cryoprotectant and neutralization of cell concentrate on freeze-drying survival, storage stability, and acid and bile exposure of Bifidobacterium animalis ssp. lactis cells produced without milkbased ingredients. J. Appl. Microbiol. 99:1330-1339.

Saarela, M., I. Virkajarvi, H. L. Alakomi, P. Sigvart-Mattila, and J. Matto. 2006. Stability and functionality of freeze-dried probiotic Bifidobacterium cells during storage in juice and milk. Int. Dairy J. $16: 1477-1482$

Scheller, M., and D. J. O'Sullivan. 2011. Comparative analysis of an intestinal strain of Bifidobacterium longum and a strain of Bifidobacterium animalis subspecies lactis in Cheddar cheese. J. Dairy Sci. 94:1122-1131.

Siaterlis, A., G. Deepika, and D. Charalampopoulos. 2009. Effect of culture medium and cryoprotectants on the growth and survival of probiotic lactobacilli during freeze-drying. Lett. Appl. Microbiol. 48:295-301.

Simpson, P. J., C. Stanton, G. F. Fitzgerald, and R. P. Ross. 2005. Intrinsic tolerance of Bifidobacterium species to heat and oxygen and survival following spray drying and storage. J. Appl. Microbiol. 99:493-501.

Stapelfeldt, H., B. R. Nielsen, and L. H. Skibsted. 1997. Effect of heat treatment, water activity and storage temperature on the oxidative stability of whole milk powder. Int. Dairy J. 7:331-339.

Wang, Y.-C., R.-C. Yu, and C.-C. Chou. 2004. Viability of lactic acid bacteria and bifidobacteria in fermented soymilk after drying, subsequent rehydration and storage. Int. J. Food Microbiol. 93:209-217.

Wong, S., B. M. Kabeir, S. Mustafa, R. Mohamad, A. S. M. Hussin, and M. Y. Manap. 2010. Viability of Bifidobacterium pseudocatenulatum G4 after spray-drying and freeze-drying. Microbiol. Insights $3: 37-43$.

Zayed, G., and Y. H. Roos. 2004. Influence of trehalose and moisture content on survival of Lactobacillus salivarius subjected to freezedrying and storage. Process Biochem. 39:1081-1086. 